\title{
Geographical Change of the Japanese National Land in the 200 Years Using INŌ's Map with GIS
}

\author{
Yuji Murayama ${ }^{\mathrm{a}}$, Yuki Iwai $^{\mathrm{b}}$ \\ ${ }^{a}$ Faculty of Life and Environmental Sciences, University of Tsukuba, 1-1-1, Tennodai, Tsukuba, Ibaraki 305-8572, Japan; \\ tmrmt@geoenv.tsukuba.ac.jp, mura@geoenv.tsukuba.ac.jp \\ ${ }^{b}$ Graduate School of Life and Environmental Sciences, University of Tsukuba, 1-1-1, Tennodai, Tsukuba, Ibaraki 305-8572, Japan; \\ s1721001@s.tsukuba.ac.jp
}

*Yuji Murayama

Keywords: INŌ's map, GIS, Japanese national land, late Edo era, spatial analysis

\begin{abstract}
:
This presentation discusses the regional changes quantitatively in the 200 years through the overlay analysis of the present map and the INŌ's map made by Tadataka INO in 1821 (Figure 1). INO surveyed the coastline and major roads on foot. He investigated not only survey lines, but also various geographic information such as rivers, lakes, mountains, village names, castles, temples, administrative boundaries, etc. Visualizing all of the 214 sheets of the INŌ's large-scale map with Geographic Information Systems (GIS), we can analyse the national land condition seamlessly at the end of the Edo era.

Methodological point of view, we have serious problems including the scale, projection, identification of geographic features and so on, when we compare the old map with the present. In this connection, digitalizing the INŌ's map as the GIS data is very useful to examine the spatial transformation scientifically during the 200 years. The digital INŌ's map was constructed by employing the geo-reference function of GIS with the triangulation method. The survey line was converted into the line feature of vector data, and the place names were converted into the point feature of raster data. The distance of the survey line was measured by GIS-based geometric operation.

We obtained the following findings. The distributions of villages, ports, and facilities in western Japan were denser than those in eastern Japan in the 19th century. This was caused not only by the difference in natural environment and landform but also by socioeconomic factors including the locations of the castle towns and industrial activities. The regional structure has been dramatically transformed by the modernization of the political system, transportation system, and industrial development in reclaimed areas (Figure 2). It is concluded that most parts of changes in regional characteristics have been attained by overcoming the natural constraints. However, the difference in the political system has also been influential to the formation of the present regional system.
\end{abstract}

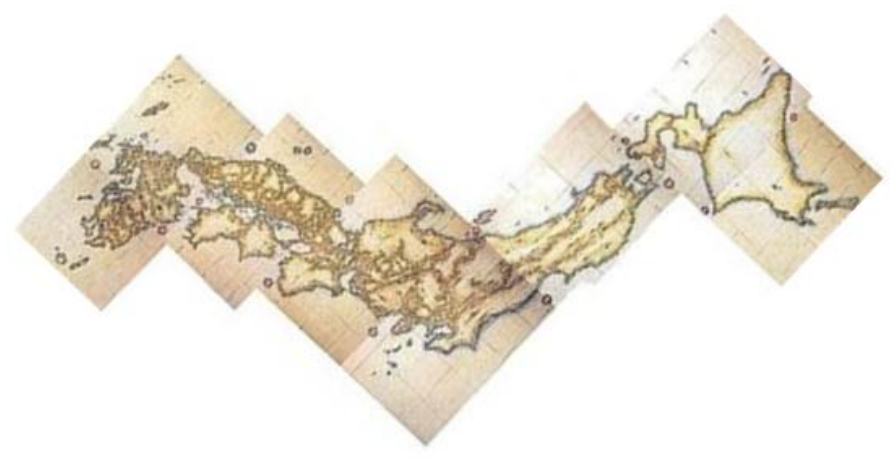

Figure 1. Map of Japan by Tadataka INO (1821) 


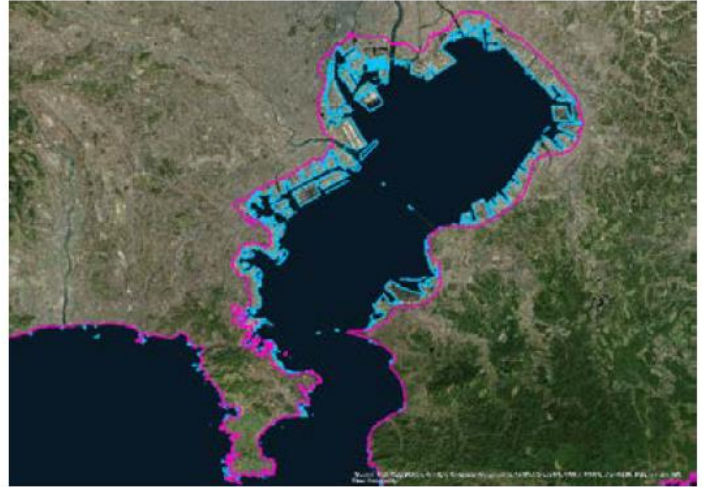

Tokyo area

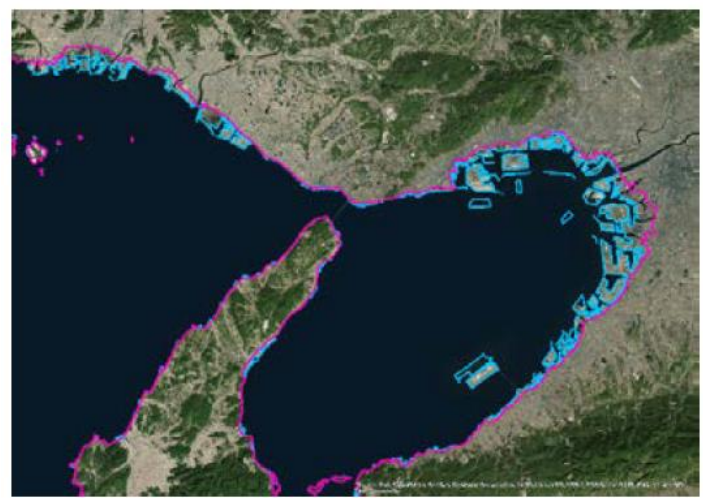

Osaka area

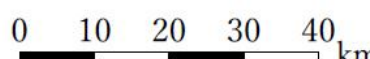

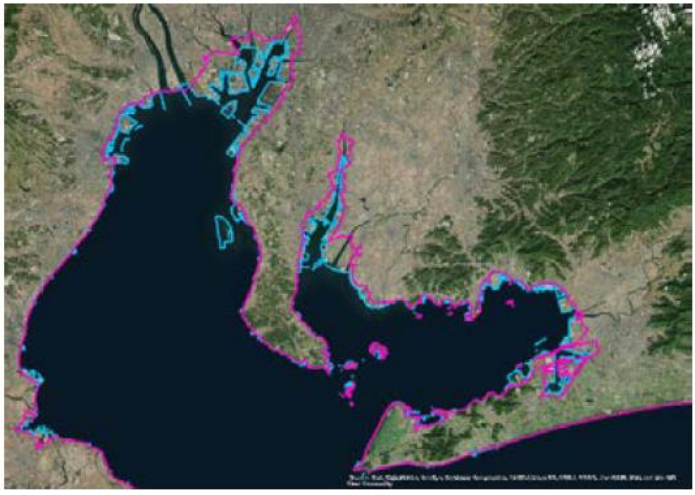

Aichi area

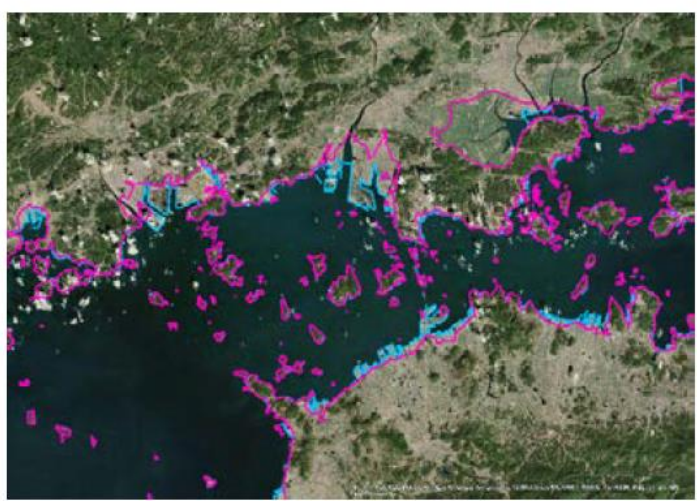

Okayama area

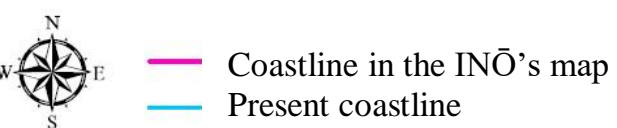

Figure 2. The coastline in the INŌ's map and the coastline in the present. 